Pesq. Vet. Bras. 30(8):659-664, agosto 2010

\title{
Doença do disco intervertebral cervical em cães: 28 casos $(2003-2008)^{1}$
}

\author{
Giancarlo Santini ${ }^{2}$, Alexandre Mazzanti ${ }^{3^{*}}$, Diego V. Beckmann², Rosmarini P. \\ Santos $^{2}$, Charles Pelizzari², Dakir Polidoro ${ }^{4}$ e Raquel Baumhardt ${ }^{4}$
}

\begin{abstract}
Santini G., Mazzanti A., Beckmann D.V., Santos R.P., Pelizzari C., Polidoro D \& Baumhardt R. 2010. [Cervical intervertebral disk disease in dogs: 28 cases (20032008).] Doença do disco intervertebral cervical em cães: 28 casos (2003-2008). Pesquisa Veterinária Brasileira 30(8):659-664. Programa de Pós-Graduação em Medicina Veterinária, Universidade Federal de Santa Maria, 97105-900 Santa Maria, RS Brazil. E-mail: alexamazza@yahoo.com.br

The aim of this study was to evaluate the medical records of dogs with cervical intervertebral disk disease (IDD), submitted to surgical treatment at the Hospital Veterinário Universitário of Universidade Federal de Santa Maria (HVU-UFSM), between January 2003 and October 2008. The animal data included breed, sex, age, neurological signs and their durations, response to surgical treatment, complications, time for functional recovery after surgery, and recurrence. All dogs showed cervical hyperaesthesia confirmed by the neck movement test. Regarding the degree of neurological dysfunction, the following were verified: degree I (eight dogs, 28.5\%), degree II (three dogs, 10.7\%), degree III (five dogs, 17.8\%), and degree V (twelve dogs, $42.8 \%$ ). In relation to the duration of neurological signs prior to surgery, seven dogs (25\%) showed neurological deficits for up to 15 days, fourteen dogs $(50 \%)$ for 15 to 30 days and the other seven dogs (25\%) showed signs of neurological deficiencies for more than 30 days. All dogs $(n=21)$ showed satisfactory recovery and no recurrence. It can be concluded that most affected dogs are middle aged, males and condrodystrophic and noncondrodystrophic breeds including large-breed dogs and the main neurological sign is cervical hyperaesthesia. The surgical treatment promotes satisfactory functional recovery and no recurrence, the main complications intraoperative are venous plexus hemorrhage, bradycardia and hypotension and the duration of clinical signs prior to surgery do not interfere with the recovery time of the dogs.
\end{abstract}

INDEX TERMS: Cervical hyperaesthesia, neurology, ventral slot, dog.

RESUMO.- O objetivo deste estudo foi identificar cães com doença do disco intervertebral (DDIV) cervical atendidos no Hospital Veterinário Universitário (HVU) da Universidade Federal de Santa Maria (UFSM) entre janeiro de 2003 e outubro de 2008 e obter informações a respeito de raça, sexo, idade, sinais neurológicos, resposta ao tratamento cirúrgico, com-

\footnotetext{
1 Recebido em 29 de outubro de 2009.

Aceito para publicação em 7 de junho de 2010.

2 Programa de Pós-Graduação em Medicina Veterinária (PPGMV), Centro de Ciências Rurais (CCR), Universidade Federal de Santa Maria (UFSM), Av. Roraima 1000, Santa Maria, RS 97105-900, Brasil.

${ }^{3}$ Departamento de Clínica de Pequenos Animais, Hospital Veterinário Universitário, UFSM, Santa Maria, RS. *Autor para correspondência: alexamazza@yahoo.com.br

${ }^{4}$ Curso de Medicina Veterinária, CCR, UFSM, Santa Maria, RS.
}

plicações, tempo de recuperação funcional após a cirurgia e ocorrência de recidiva. Hiperestesia cervical foi observada em todos os cães $(n=28)$. Quanto ao grau de disfunção neurológica foram verificados: grau I (8/28[28,5\%]), grau II (3/28 $[10,7 \%])$, grau III (5/28[17,8\%]) e grau V (12/2 [42,8\%]). A duração dos sinais neurológicos antes da cirurgia em sete cães (25\%) permaneceu por até 15 dias, em 14 cães $(50 \%)$ entre 15 e 30 dias e nos outros sete cães $(25 \%)$ por mais de 30 dias. A recuperação satisfatória e sem recidiva foi observada em todos os cães submetidos ao tratamento cirúrgico e que sobreviveram $(n=21)$. Pode-se concluir que a DDIV cervical em nossa rotina acomete principalmente cães adultos, machos, de raças condrodistróficas e não condrodistróficas, incluindo as de grande porte; a hiperestesia cervical é a principal manifestação clínica; a técnica de fenda ventral promo- 
ve recuperação funcional satisfatória e sem recidiva; as principais complicações trans-operatórias são a hemorragia do plexo venoso, a bradicardia e a hipotensão; e a duração dos sinais clínicos não interfere no tempo de recuperação pósoperatória dos cães.

TERMOS DE INDEXAÇÃO: Hiperestesia cervical, neurologia, fenda ventral, cão.

\section{INTRODUÇÃO}

Doença do disco intervertebral (DDIV) é a condição neurológica mais frequentemente descrita em cães (Toombs 1992, McCartney 2007). A região cervical representa aproximadamente $15 \%$ dos casos de DDIV (Lemarié et al. 2000). Essa alteração ocorre em raças condrodistróficas e não condrodistróficas (Toombs 1992, De Lahunta \& Glass 2009), ocasionando extrusão (Hansen tipo I) ou protrusão (Hansen tipo II) do disco degenerado no interior do canal vertebral (Toombs \& Bauer 1998, Sharp \& Wheeler 2005).

$\mathrm{O}$ disco entre as vértebras cervicais $\mathrm{C} 2 / \mathrm{C} 3$ é o de maior ocorrência da DDIV e essa prevalência diminui caudalmente (Dalmann et al. 1992, Toombs 1992, Sharp \& Wheeler 2005, Itoh et al. 2008). O segmento $\mathrm{C} 6 / \mathrm{C} 7$ é raramente afetado, com exceção de cães de raças de grande porte, como parte da espondilomielopatia cervical caudal (Sharp \& Wheeler 2005).

A hiperestesia cervical é frequentemente observada na compressão da medula espinhal cervical em cães com DDIV. Outros sinais clínicos incluem disfunção motora como ataxia proprioceptiva, hemiparesia, tetraparesia (Toombs 1992) e claudicação devido à compressão de raiz nervosa (Seim \& Prata 1982, Sharp \& Wheeler 2005, Toombs \& Waters 2007).

O diagnóstico da DDIV cervical baseia-se na resenha, no histórico clínico, no exame neurológico (Toombs \& Waters 2007), na radiografia simples e contrastada (mielografia), na tomografia computadorizada, na mielotomografia (Itoh et al. 2008) e na ressonância magnética nuclear (Sharp \& Wheeler 2005). A principal confirmação da doença ocorre durante a cirurgia descompressiva (Toombs \& Waters 2007), quando é observado o conteúdo do disco no interior do canal vertebral (extrusão) ou a projeção do anel fibroso (protrusão) ocasionando compressão da medula espinal (Braund 1996, Sharp \& Wheeler 2005).

Os métodos de tratamento em cães com DDIV cervical, incluem cirurgia descompressiva ou tratamento clínico conservativo composto por confinamento e utilização de antiinflamatórios. Diversas técnicas cirúrgicas têm sido descritas, incluindo descompressão por fenda (slot) ventral, fenestração de DDIV ventral, laminectomia descompressiva por acesso lateral ou dorsal (Sharp \& Wheeler 2005, Toombs \& Waters 2007), hemilaminectomia (Tanaka et al. 2005) e facetectomia (Fossum 2007).

A maioria dos cães responde satisfatoriamente ao tratamento conservativo. As desvantagens deste tratamento incluem a recidiva, que pode chegar a 33\% (Toombs 1992), além do tempo que o cão demora a se restabelecer (Russell \& Griffiths 1968). As indicações para o tratamento cirúrgi- co da DDIV cervical são a falta de resposta ao tratamento conservativo, deficiências neurológicas marcantes e progressivas e hiperestesia cervical persistente (Toombs \& Waters 2007, Sharp \& Wheeler 2005, Fossum 2007). As desvantagens incluem morte durante o procedimento anestésico, deterioração neurológica e possível subluxação vertebral (McCartney 2007).

Vários trabalhos sobre DDIV cervical em cães foram publicados no exterior. No Brasil, poucos estudos foram divulgados sobre a doença, despertando o interesse sobre o assunto. Diante disso, o objetivo deste estudo retrospectivo foi identificar cães com DDIV cervical atendidos no Hospital Veterinário Universitário (HVU) da Universidade Federal de Santa Maria (UFSM) entre janeiro de 2003 e outubro de 2008 e obter informações a respeito de raça, sexo, idade, sinais neurológicos e sua duração, resposta ao tratamento cirúrgico, complicações, tempo de recuperação funcional após a cirurgia e ocorrência de recidiva.

\section{MATERIAL E MÉTODOS}

Foram analisados os registros médicos de cães atendidos pelo serviço de neurologia do HVU/UFSM entre janeiro de 2003 e outubro de 2008 que apresentavam sinais neurológicos sugestivos de DDIV cervical. Os critérios de inclusão foram os casos de DDIV cervical que apresentavam exame neurológico completo, exame radiográfico simples e contrastado (mielografia) e tratamento cirúrgico.

A DDIV cervical foi confirmada durante a cirurgia descompressiva, em que foram removidos conteúdos do núcleo pulposo e/ou anel fibroso (extrusão de disco) ou notada a projeção do anel fibroso (protrusão) para o interior do canal vertebral. Informações gerais sobre raça, sexo, idade, sinais neurológicos e sua duração, resposta ao tratamento cirúrgico, complicações, tempo de recuperação funcional após a cirurgia e recidiva foram obtidos.

O exame neurológico foi realizado em todos os cães e incluía a avaliação sobre: estado mental, postura e locomoção, reações posturais, avaliação dos nervos cranianos e reflexos segmentares da medula espinhal. A hiperestesia cervical foi confirmada por palpação e pela movimentação voluntária do pescoço em flexão, extensão e lateralidade durante o fornecimento de alimentos, conforme Costa et al. (2006).

As alterações neurológicas foram classificadas em graus, conforme citação de Costa \& Parent (2007), sendo: grau I somente hiperestesia cervical; grau II - ataxia ou paresia leve do membro pélvico com ou sem envolvimento do membro torácico; grau III - ataxia ou paresia moderada do membro pélvico com envolvimento do membro torácico; grau IV - ataxia e paresia marcante do membro pélvico com envolvimento do membro torácico e; grau V - tetraparesia não-ambulatória.

O exame radiográfico contrastado foi realizado em quatro posições compreendidas em látero-lateral, ventro-dorsal e ventro-dorsal oblíqua esquerda e direita numa angulação de $45^{\circ}$, com a finalidade de localizar o espaço acometido e verificar a existência de lateralidade da compressão medular. Os cães foram submetidos à anestesia geral, para a realização da mielografia (Ducoté \& Dewey 2006). O acesso para a injeção do contraste foi através da cisterna magna conforme técnica descrita por Sharp \& Wheeler (2005). Os cães que apresentavam linha de contraste caracterizando compressão extramedular, su- 
gerindo DDIV cervical foram submetidos à cirurgia descompressiva através da fenda (slot) ventral (Sharp \& Wheeler 2005).

Decorridos doze meses do procedimento cirúrgico, os proprietários dos cães com DDIV cervical foram contatados por telefone na tentativa de obter-se resposta quanto à recuperação sendo considerada satisfatória (ausência de hiperestesia cervical e caminhar sem quedas) ou insatisfatória (presença de hiperestesia cervical e caminhar com quedas) e quanto à recidiva dos sinais clínicos (ocorrência ou não ocorrência). $O$ tempo da duração dos sinais clínicos e da recuperação foi definido como: $<15$ dias, entre 15-30 dias e >30 dias e; $<7$ dias, entre 7 e 15 dias e $>15$ dias, respectivamente.

A relação entre a duração dos sinais clínicos e o tempo de recuperação foi analisada pelo teste de Fisher $(P<0,05)$.

\section{RESULTADOS E DISCUSSÃO}

Foram incluídos nesse estudo 28 cães com DDIV cervical, resultando uma média de 5,6 casos/ano. Já Dallman et al. (1992) citaram a ocorrência de 105 casos em dez anos (10,5 casos/ano), Forterre et al. (2008) realizaram levantamento dos registros médicos durante o período de 2004 a 2007 e encontraram 35 cães ( 11,6 casos/ano). Itoh et al. (2008) observaram 132 casos (10,1 casos/ano) entre 1991 e 2004. Diversos fatores provavelmente influenciaram no número reduzido de cães neste trabalho como a responsividade da doença ao tratamento clínico, principalmente para cães em grau I de disfunção neurológica, o risco anestésico-cirúrgico e o custo da cirurgia.
A idade média dos cães com DDIV cervical foi de 7,8 $( \pm 3,01)$ anos (Quadro 1). Em um estudo realizado por Cudia \& Duval (1997), foi verificado que a extrusão de disco ocorreu em cães condrodistróficos com idade entre dois e sete anos (pico de incidência entre quatro e cinco anos) e a protrusão de disco em cães não condrodistróficos com idade entre oito e dez anos. Neste estudo, dos 25 cães que tiveram extrusão de disco, $14(56 \%)$ eram de raças não condrodistróficas, sendo cinco $(35,7 \%)$ com idade entre quatro e sete anos. Cherrone et al. (2004) encontraram DDIV cervical Hansen tipo I em 46 cães de raças não condrodistrófica de médio e grande porte com uma média de idade de 7,8 anos. Logo, os resultados demonstraram que se deve incluir a Hansen tipo I em cães com suspeita de DDIV cervical, mesmo que sejam de raças não condrodistrófica, incluindo as de grande porte.

Neste trabalho, 17 cães $(60,7 \%)$ acometidos por DDIV cervical eram machos (Quadro 1). Itoh et al. (2008) também encontraram maior incidência nos machos e citaram a possibilidade de haver influência de hormônio sexual neste tipo de doença. Já Dallman et al. (1992) observaram uma maior prevalência em fêmeas e Somerville et al. (2001) descreveram que ambos os sexos apresentaram risco de serem acometidos. Nenhuma correlação foi demonstrada até o presente momento entre a predisposição sexual e a DDIV cervical.

O predomínio da raça com DDIV cervical (Quadro 1), neste estudo, foi o Dachshund, corroborando com as cita-

Quadro 1. Representação dos 28 cães atendidos no HVU/UFSM no período de 2003 a 2008 com doença do disco intervertebral cervical

\begin{tabular}{|c|c|c|c|c|c|c|c|}
\hline $\begin{array}{c}\text { Raça, sexo, idade, ano } \\
\text { de atendimento }\end{array}$ & $\begin{array}{l}\text { Local de } \\
\text { compressão }\end{array}$ & $\begin{array}{l}\text { Duração de } \\
\text { Sinais clínicos }\end{array}$ & $\begin{array}{c}\text { Tipo de } \\
\text { compressão }\end{array}$ & $\begin{array}{l}\text { Grau de } \\
\text { disfunção }\end{array}$ & $\begin{array}{l}\text { Recuperação } \\
\text { funcional }\end{array}$ & $\begin{array}{l}\text { Tempo de } \\
\text { recuperação }\end{array}$ & Recidiva \\
\hline SRD, M, 7 anos, 2003 & C3-C4 & 11 dias & Extrusão & III & Satisfatória & 8 dias & Não \\
\hline Dachshund, F, 6 anos, 2003 & C3-C4 & 3 dias & Extrusão & III & Satisfatória & 5 dias & Não \\
\hline Poodle, M, 13 anos, 2003 & C3-C4 & 4 dias & Extrusão & III & Satisfatória & 15 dias & Não \\
\hline SRD, F, 4 anos, 2004 & C4-C5 & 30 dias & Extrusão & III & Satisfatória & 3 dias & Não \\
\hline Dachshund, F, 5 anos, 2004 & C2-C3 & 15 dias & Extrusão & 1 & Satisfatória & 6 dias & Não \\
\hline SRD, M, 5 anos, 2004 & C4-C5 & 40 dias & Extrusão & V & Satisfatória & 21 dias & Não \\
\hline Dachshund, M, 7 anos, $2004^{*}$ & C4-C5 & 21 dias & Extrusão & V & - & - & - \\
\hline Dobermann, M, 8 anos, 2004* & $\mathrm{C} 6-\mathrm{C} 7$ & 25 dias & Protrusão & V & - & - & - \\
\hline SRD, M, 5 anos, 2005 & C5-C6 & 17 dias & Extrusão & II & Satisfatória & 3 dias & Não \\
\hline Dobermann, M, 8 anos, 2005* & C3-C4 & 21 dias & Extrusão & V & - & - & - \\
\hline Dachshund, M, 10 anos, 2006 & C2-C3 & 33 dias & Extrusão & I & Satisfatória & 5 dias & Não \\
\hline Beagle, F, 5 anos, 2006 & C4-C5 & 22 dias & Extrusão & I & Satisfatória & 7 dias & Não \\
\hline Dachshund, F, 4 anos, 2006 & C3-C4 & 3 dias & Extrusão & II & Satisfatória & 7 dias & Não \\
\hline SRD, M, 15 anos, $2006^{*}$ & $\mathrm{C} 3-\mathrm{C} 4$ & 17 dias & Extrusão & V & - & - & - \\
\hline Poodle, F, 8 anos, $2006^{*}$ & C5-C6 & 14 dias & Extrusão & V & - & - & - \\
\hline Cocker, F, 6 anos, 2007 & $\mathrm{C} 2-\mathrm{C} 3$ & 60 dias & Extrusão & 1 & Satisfatória & $1 \mathrm{dia}$ & Não \\
\hline Dachshund, M, 7 anos, 2007 & C3-C4 & 90 dias & Extrusão & I & Satisfatória & 21 dias & Não \\
\hline Rottweiler, F, 9 anos, 2007 & C3-C4 & 52 dias & Extrusão & V & Satisfatória & 21 dias & Não \\
\hline Beagle, M, 5 anos, 2008 & $\mathrm{C} 2-\mathrm{C} 3$ & 22 dias & Extrusão & $\mathrm{I}$ & Satisfatória & 7 dias & Não \\
\hline Cocker Spaniel, F, 7 anos, 2008 & C3-C4 & 60 dias & Extrusão & I & Satisfatória & 6 dias & Não \\
\hline Cocker Spaniel, F, 8 anos, 2008 & $\mathrm{C} 3-\mathrm{C} 4$ & 24 dias & Extrusão & 1 & Satisfatória & 5 dias & Não \\
\hline Dachshund, M, 9 anos, 2008 & C3-C4 & 30 dias & Extrusão & II & Satisfatória & 3 dias & Não \\
\hline Dobermann, M, 8 anos, 2008 & $\mathrm{C} 6-\mathrm{C} 7$ & 120 dias & Protrusão & III & Satisfatória & 7 dias & Não \\
\hline Dachshund, M, 8 anos, 2008 & C3-C4 & 21 dias & Extrusão & V & Satisfatória & 5 dias & Não \\
\hline Dachshund, M, 14 anos, 2008 & C4-C5 & 4 dias & Extrusão & V & Satisfatória & 15 dias & Não \\
\hline Dachshund, F, 5 anos, 2008 & C3-C4 & 30 dias & Extrusão & V & Satisfatória & 3 dias & Não \\
\hline Dachshund, M, 12 anos, 2008* & C4-C5 & 3 dias & Extrusão & V & - & - & - \\
\hline Dobermann, M, 12 anos, $2008^{*}$ & $\mathrm{C} 6-\mathrm{C} 7$ & 30 dias & Protrusão & V & - & - & - \\
\hline
\end{tabular}

a $\mathrm{M}=$ macho, $\mathrm{F}$ = fêmea, $\mathrm{SRD}=$ sem raça definida, ${ }^{*}$ morte. 
ções de Funkquist \& Svalastoga (1979), Swaim \& Hyam (1982), Dallman et al. (1992), Gill et al. (1996), Fitch et al. (2000) e Cherrone et al. (2004). Por outro lado, Itoh et al. (2008) verificaram o Beagle e justificaram a maior incidência devido à preferência dessa raça no Japão, quando comparado a outros países. Mesmo havendo maior predisposição em raças condrodistróficas, verificou-se, neste estudo, que a DDIV cervical pode acometer cães de diferentes raças consideradas ou não condrodistróficas.

A maneira empregada para avaliar a hiperestesia cervical nos cães deste estudo foi de acordo com as indicações de Costa et al. (2006), sendo eficaz em 100\% dos casos. A movimentação do pescoço realizada passivamente e de maneira exagerada em diferentes posições pode ocasionar danos neurológicos adicionais, conforme salientado por Sharp \& Wheeler (2005).

O disco intervertebral mais acometido foi entre C3-C4 (Fig.1), ao contrário do observado por Dalmann et al. (1992), Toombs (1992), Sharp \& Wheeler (2005) e Itoh et al. (2008) que verificaram maior incidência entre C2-C3. Adicionalmente, a mielografia revelou, em todos os cães, o envolvimento de um único disco intervertebral, ao contrário dos estudos

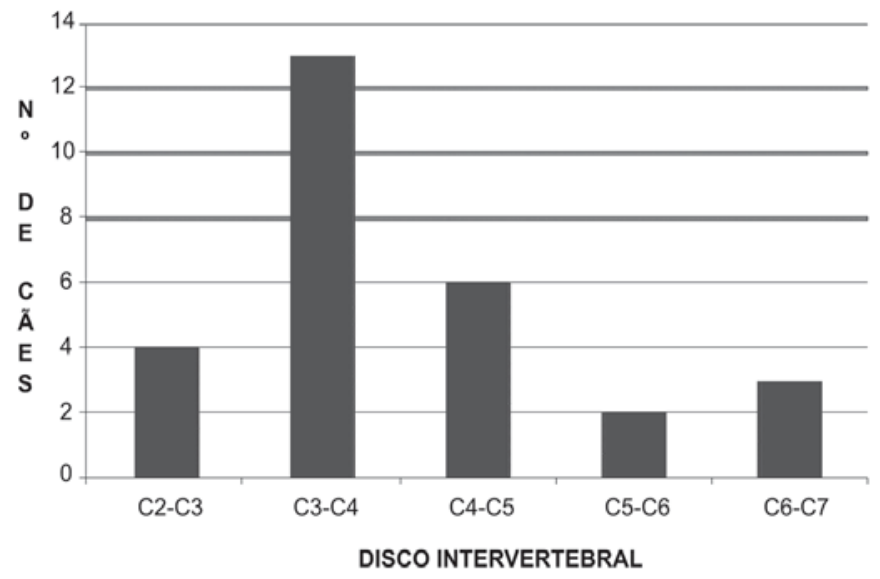

Fig.1. Representação esquemática do número de casos em relação ao local afetado em cães com DDIV cervical.

de Fitch et al. (2000) e Tanaka et al. (2005) que observaram o envolvimento de mais de um disco intervertebral ocasionando sinais clínicos da doença. Em três cães, o disco acometido estava entre $\mathrm{C} 6-\mathrm{C} 7$ e todos apresentavam espondilomielopatia cervical caudal coincidindo com os achados de Sharp \& Wheeler (2005) ao mencionarem que este local é raramente afetado, com exceção de cães de raças de grande porte, como parte dessa doença.

Neste estudo, cinco cães que estavam em grau I, dois em grau II, dois em grau III e dois cães em grau V de disfunção neurológica tiveram melhora dos sinais clínicos antes dos sete dias do procedimento cirúrgico (Fig.2 e 4). Outros seis cães $(21,5 \%)$ recuperaram de forma satisfatória entre sete e 15 dias, sendo que, dois estavam em grau I, um em grau II, dois em grau III e um em grau V. Os três cães restantes (um em grau I e dois em V), o tempo de

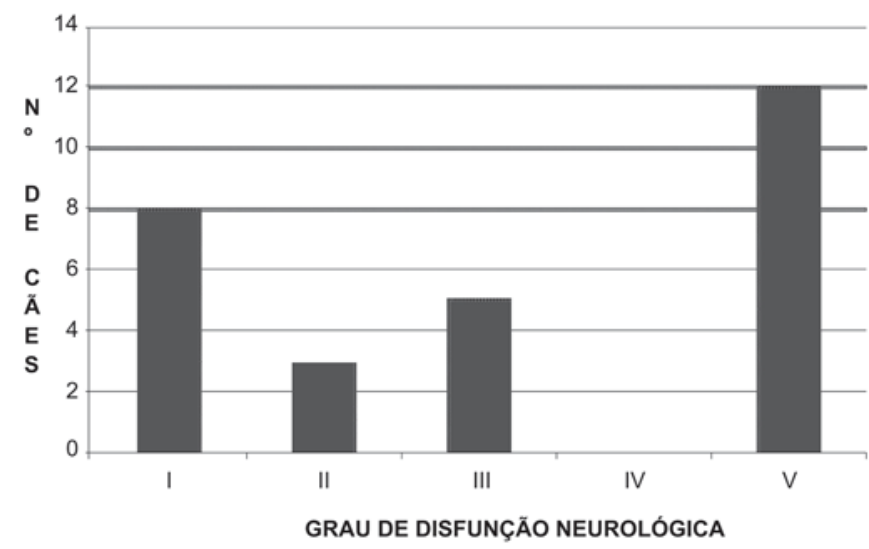

Fig.2. Representação esquemática do grau de disfunção neurológica de cães afetados pela DDIV cervical.

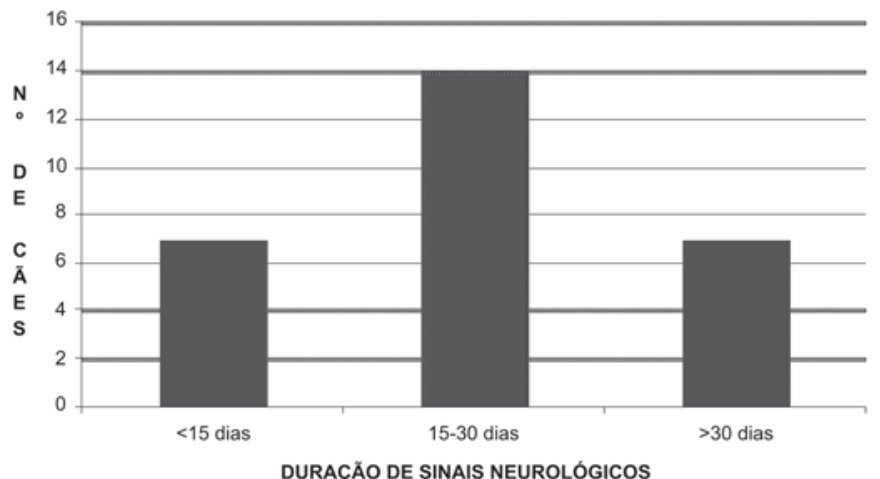

Fig.3. Representação esquemática da duração dos sinais neurológicos de cães afetados pela DDIV cervical.

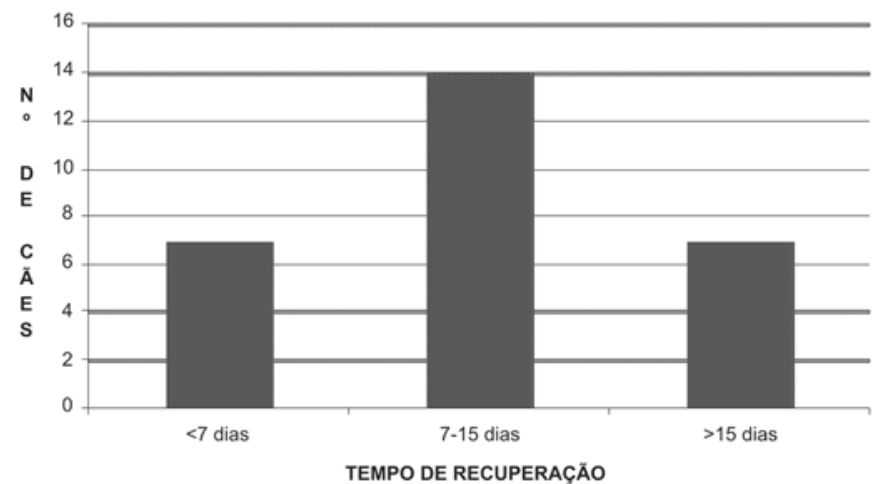

Fig.4. Representação esquemática do tempo de recuperação dos sinais neurológicos de cães afetados pela DDIV cervical após o tratamento cirúrgico.

recuperação foi superior a 15 dias. Pode-se verificar, com isso, que $50 \%$ dos cães tiveram recuperação funcional satisfatória em um tempo inferior a sete dias, independente do estágio de disfunção neurológica. Seim \& Prata (1982) quando submeteram cães à cirurgia descompressiva cervical com diferentes sinais de disfunção neurológica, ou seja, somente hiperestesia cervical, tetraparesia ambulatória e não ambulatória também observaram, dentro de sete dias, uma melhora clinica de $73 \%, 71 \%$ e $100 \%$, respecti- 
vamente, voltando os mesmos a deambular neste período e sem sinais de hiperestesia.

Sete cães $(25 \%)$ permaneceram por até 15 dias com deficiência neurológica antes da cirurgia, destes, um apresentou tempo de recuperação inferior a sete dias e os outros quatro recuperaram entre sete e 15 dias. Já 14 cães (50\%) tiveram duração dos sinais clínicos entre 15 e 30 dias e, com exceção de seis cães que morreram no transoperatório, cinco recuperaram com menos de sete dias e três entre 7 e 15 dias. Outros sete cães (25\%) permaneceram por mais de 30 dias com deficiências neurológicas, sendo que três recuperaram em menos de sete dias, um entre sete e quinze dias e três com mais de quinze dias (Fig.3 e 4). A relação entre duração dos sinais clínicos antes da cirurgia e o tempo para recuperação não apresentou diferença estatística significativa, corroborando com Kazakos et al. 2005 que avaliou a duração e severidade dos sinais clínicos como indicadores de prognóstico e não foi possível associá-los. No entanto, Tanaka et al. (2005) que avaliaram 18 cães com DDIV cervical, observaram que dois cães que tiveram um longo tempo de duração de sinais clínicos antes da cirurgia levaram mais tempo para recuperar.

Os vinte e oito cães deste estudo que foram submetidos primeiramente ao tratamento clínico não tiveram melhora, optando-se pelo procedimento cirúrgico, sendo considerado satisfatório em todos os casos em que não houve morte durante o procedimento $(n=21)$. A falta de resposta ao tratamento clínico pode ser atribuída ao uso de anti-inflamatórios sem a realização de repouso absoluto, tornando a doença de evolução crônica e progressiva. Em três cães deste estudo que apresentavam duração dos sinais clínicos de 60,90 e 120 dias, respectivamente, tiveram piora transitória dos sinais neurológicos no pós-operatório provavelmente pela manipulação excessiva da medula espinhal em decorrência da aderência do material do disco à dura-máter e ao plexo venoso. Com isso, verifica-se que cães com persistência dos sinais clínicos poderão apresentar uma maior chance complicações intra-operatórias.

Foi observado em $82,1 \%$ dos cães $(n=28)$ deste estudo hemorragia do plexo venoso, considerada uma das principais complicações no transoperatório ao empregar-se a técnica de fenda ventral (Sharp \& Wheeler 2005). Seguindo as recomendações de Fossum (2007) e Toombs \& Waters (2007) foram utilizados fragmentos musculares retirados das proximidades do leito cirúrgico e colocados temporariamente sobre os locais de sangramento o que permitiu o controle da hemorragia em todos dos casos. Já Tanaka et al. (2005), no entanto, não encontraram excessiva hemorragia perioperatório durante a cirurgia descompressiva da medula espinhal de cães com DDIV cervical.

Um cão que se encontrava em grau $V$ de disfunção neurológica (tetraparesia não-ambulatória) foi submetido à cirurgia descompressiva entre os discos cervicais C4-C5 e fenestração de disco entre C3-C4. Porém, o mesmo continuou a apresentar hiperestesia cervical após uma semana do procedimento cirúrgico. Na mielografia pós-operatória, não ficou evidenciada compressão medular na região do disco fenestrado (C3-C4), o que descartou outra cirurgia, optando-se pelo tratamento clínico. Após uma semana, o cão obteve melhora dos sinais clínicos neurológicos, sendo considerado satisfatório a sua recuperação funcional. Embora Toombs \& Waters (2007), mencionaram a necessidade de reintervenção cirúrgica em cães submetidos à fenestração isolada de disco, os sinais persistentes no cão desta pesquisa corroboraram com os achados de Sharp \& Wheeler (2005) ao mencionarem a possibilidade de persistência de hiperestesia cervical no pós-operatório de cães submetidos à fenestração podendo levar até 60 dias para desaparecer.

Foi verificado morte em $25 \%$ dos cães $(n=7)$ no transoperatório em decorrência de bradicardia aguda e hipotensão não responsiva ao tratamento farmacológico. Todos se encontravam em grau $\mathrm{V}$ de disfunção neurológica e, em três deles (50\%), a DDIV estava associada à cardiomiopatia dilatada. Estes achados corroboraram com Sharp \& Wheeler (2005) que relataram um aumento na taxa de mortalidade para cães em tetraparesia não ambulatória antes da cirurgia. Os resultados também encontrados nesta pesquisa demonstraram o aumento do risco anestésico-cirúrgico para cães com grave deterioração neurológica, principalmente com doenças pré-existentes, o que pode auxiliar o cirurgião na emissão do prognóstico perante o proprietário e da importância da monitoração da pressão arterial, da frequência cardíaca e respiratória.

\section{CONCLUSÕES}

Com base nas informações obtidas pode-se concluir que:

DDIV cervical em nossa rotina acomete cães adultos, machos, de raças condrodistróficas e não condrodistróficas, incluindo as de grande porte;

hiperestesia cervical é a manifestação clínica mais frequente;

técnica cirúrgica de fenda ventral promove recuperação funcional satisfatória sem ocasionar recidiva;

as principais complicações trans-operatórias são a hemorragia do plexo venoso, bradicardia e hipotensão; e

a duração dos sinais clínicos não interfere no tempo de recuperação pós-operatória.

Agradecimentos.- Aos técnicos em Radiologia Antonio César de Oliveira Guerra e Daves Pereira Filho do HVU/UFSM pelo auxílio na realização das radiografias simples e da mielografia e à Coordenação de Aperfeiçoamento de Pessoal de Nível Superior (CAPES), pela concessão de bolsa de mestrado.

\section{REFERÊNCIAS}

Braund K.B. 1996. Traumatismo agudo da medula espinhal, p.13111326. In: Bojrab M.J (Ed.), Mecanismos da Moléstia na Cirurgia de Pequenos Animais. $2^{\mathrm{a}}$ ed. Manole, São Paulo.

Cherrone K.L., Dewey C.W., Coates J.R. \& Bergman R.L. 2004. A retrospective comparison of cervical intervertebral disk disease in nonchondrodystrophic large dogs versus small dogs. J. Am. Anim. Hospital Assoc. 40:316-320.

Costa R.C., Parent J., Dobson H., Holmberg D. \& Partlow G. 2006. Comparison of magnetic resonance imaging and myelography in 18 Doberman Pinscher dogs with cervical spondylomyelopathy. Vet. Radiol. Ultrasound. 47(6):523-531. 
Costa R.C. \& Parent J.M. 2007. One-year clinical and magnetic resonance imaging follow-up of Doberman Pinschers with cervical spondylomyelopathy treated medically or surgically. J. Am. Vet. Med. Assoc. 231:243-250.

Dallman M.J., Palettas P. \& Bojrab M.J. 1992. Characteristics of dogs admitted for treatment of cervical intervertebral disk disease: 105 cases (1972-1982). J. Am. Vet. Med. Assoc. 200:2009-2011.

De Lahunta A. \& Glass E. 2009. Veterinary Neuroanatomy and Clinical Neurology. $3^{\text {rd }}$ ed. W.B. Saunders, St Louis, p.243-284.

Ducoté J.M. \& Dewey C.W. 2006. Diagnóstico das doenças do sistema nervoso, p.35-58. In: Dewey C.W. (Ed.), Neurologia de Cães e Gatos. Roca, São Paulo.

Fitch R.B., Kerwin S.C. \& Hosgood G. 2000. Caudal cervical intervertebral disk disease in the small dog: Role of distraction and stabilization in ventral slot decompression. J. Am. Anim. Hospital Assoc. 36:68-74.

Forterre F., Konar M., Tomek A., Doherr M., Howard J., Spreng D., Vandevelde M. \& Jaggy A. 2008. Accuracy of the withdrawal reflex for localization of the site of cervical disk herniation in dogs: 35 cases (2004-2007). J. Am. Vet. Med. Associ. 232:559-563.

Fossum T.W. 2007. Small Animal Surgery. $3^{\text {rd }}$ ed. Elsevier, St Louis, p.1412-1459.

Funkquist B. \& Svalastoga E. 1979. A simplified surgical approach to the last two cervical dises of the dog. J. Small Anim. Pract. 20:593601.

Gill P.J., Lippincott C.L. \& Anderson S.M. 1996. Dorsal laminectomy in the treatment of cervical intervertebral disk disease in small dogs: A retrospective study of 30 cases. J. Am. Anim. Hospital Assoc. 32:7780.

Itoh H., Hara Y., Yoshimi N., Harad Y., Nezu Y., Yogo T., Ochi H., Hasegawa D., Orima H. \& Tagawa M. 2008. A retrospective study of intervertebral disc herniation in dogs in Japan: 297 cases. J. Vet. Med. Sci. 70:701-706.

Kazakos G., Polizopoulou Z.S., Patsikas M.N., Tsimopoulos G., Roubies $\&$ Dessiris A. 2005. Duration and severity of clinical signs as prognostic indicators in 30 dogs with thoracolumbar disk disease after surgical descompression. J. Vet. Med. Assoc. 52:147-152.

Lemarié R.J., Kerwin B.P., Partington B.P. \& Hosgood G. 2000. Vertebral subluxation following ventral cervical decompression in the dog. J. Am. Anim. Hospital Assoc. 36:348-358.

McCartney W. 2007. Comparison of recovery times and complications rates between a modified slanted slot and the standart ventral slot for the treatment of cervical disc disease in 20 dogs. J. Small Anim. Pract. 48:498-501.

Russell S.W. \& Griffiths R.C. 1968. Recurrence of cervical disc syndrome in surgically and conservatively treated dogs. J. Am. Vet. Med. Assoc. 153:1412-1417.

Seim H.B. \& Prata R.G. 1982. Ventral decompression for treatment of cervical disk disease in the dog: A review of 54 cases. J. Am. Anim. Hospital Assoc. 18:233-240.

Somerville M.E., Anderson S.M., Gill P.J., Kantrowitz B.J. \& Stowater J.L. 2001. Accuracy of localization of cervical intervertebral disk extrusion or protrusion using survey radiography in dogs. J. Am. Anim. Hospital Assoc. 37:563-572.

Swaim S.F. \& Hyams D. 1982. Clinical observations and client evaluation of ventral decompression for cervical intervertebral disk protrusion. J. Am. Vet. Med. Assoc. 181:259-260.

Tanaka H., Nakayama M. \& Takase K. 2005. Usefulness of hemilaminectomy for cervical intervertebral disk disease in small dogs. J. Vet. Med. Sci. 67:679-683.

Toombs J.P. 1992. Cervical intervertebral disk disease in dogs. Compend. Cont. Educ. Pract. Vet. 14:1477-1488.

Toombs J.P. \& Bauer M.S. 1998. Afecção do disco intervertebral, p.1287-1305. In: Slatter D. (Ed.), Manual de Cirurgia de Pequenos Animais. $2^{\mathrm{a}}$ ed. Manole, São Paulo.

Toombs J.P. \& Waters D.J. 2007. Intervertebral disc disease, p.11931208. In: Slatter D. (Ed.), Textbook of Small Animal Surgery. $3^{\text {rd }}$ ed. Elsevier, Philadelphia.

Sharp N.J.H. \& Wheeler S.J. 2005. Small Animal Spinal Disorders: Diagnosis and surgery. $2^{\text {nd }}$ ed. Elsevier Mosby, Philadelphia, p.121-159. 